(fasting and post-glucose load) and daily activity (Actigraph ${ }^{\mathrm{TM}}$ and Actilife $^{\mathrm{TM}}$ software). We measured 31-P and 1-H magnetic resonance spectroscopy (MRS) and assessed intra-hepatic lipid (IHL) content and phospho-creatine recovery after standardised exercise within the MR scanner.

Results IHL was associated with increases in body mass and fat mass index ( $\%$ body fat $/$ height $^{2}$ ). There was a weak association between glucose levels and muscle recovery time with increased IHL. Recovery from exercise was correlated with \% time spent in daily moderate-to-vigorous physical activity (MVPA) and sedentary activity. Only 5 children achieved an activity time within $10 \%$ of the recommended 60 minutes or more of MVPA per day (mean:39 minutes).

Conclusions Children born preterm have evidence of adverse metabolic outcomes in later life. IHL deposition is related to overall fatness, and may be significant in adverse metabolic processes. Measured physical activity correlates with the ability of muscle to recover from a defined exercise. Improving MVPA may result in health benefits.

\section{EVALUATION OF THE "FREGGIE FRIDAY" PROGRAM TO PROMOTE FRUIT AND VEGETABLE CONSUMPTION IN CANADIAN ELEMENTARY SCHOOL-AGED CHILDREN}

doi:10.1136/archdischild-2012-302724.1438

1,2KB Adamo, ' $\mathrm{C}$ Colapinto, ' $\mathrm{A}$ Harvey, ${ }^{1} \mathrm{KP}$ Grattan, ${ }^{3} \mathrm{~N}$ Barrowman, $1.4,5 \mathrm{GS}$ Goldfield. 'Healthy Active Living and Obesity Research Group, Children's Hospital of Eastern Ontario (CHEO), Research Institute; 'Human Kinetics, University of Ottawa; ${ }^{3}$ Children's Hospital of Eastern Ontario (CHEO), Research Institute; ${ }^{4}$ Human Kinetics and Psychology, University of Ottawa; ${ }^{5}$ Psychology, Carleton University, Ottawa, ON, Canada

Background While Canada is one of the world's most prosperous nations, the health of our children is dismal, with obesity rates amongst the highest in the world. A healthy diet, including at least 5 to 6 daily servings of fruit and vegetables, is of profound importance to child health.

Aim To evaluate the efficacy of a fruit and vegetable program 'Freggie Fridays' developed to encourage Canadian elementary school children (grades 1 to 6 ) to eat the recommended number of fruit and vegetable servings each day.

Methods A prospective quasi-experimental trial compared schools receiving the "Freggie Friday" curriculum as the intervention $(n=8)$ to those not receiving the curriculum as control $(n=6)$. The primary outcome measure was the difference in levels of fruit and vegetable consumption as measured by a food frequency questionnaire. Information on attitudes and knowledge of fruit and vegetable consumption was attained using an adapted version of the validated Pro-Children study questionnaire.

Results A total of 807 of the 942 children who completed the baseline questionnaires completed the follow-up questionnaires (450 intervention and 357 control). A mixed effects regression model indicated no significant intervention effects on fruit or vegetable consumption, snack food consumption, or knowledge or attitudes relating to fruit and vegetable consumption.

Conclusions Despite clear messaging and a sound program, it appears that adding a nutritional program, which expects busy teachers to add this to their educational curriculum, may not be the most efficacious method of eliciting healthy dietary behaviour change in Canadian elementary school-aged children.

\section{OBESITY STRUCTURAL LINK: PARENTING STRESS, FEEDING AND EATING IMPACT ON CHINESE YOUNG CHILDREN WEIGHT OUTCOMES IN HONG KONG}

doi:10.1136/archdischild-2012-302724.1439
CMS Chan, JHM Lam. Department of Psychological Studies, The Hong Kong Institute of Education, Hong Kong, Hong Kong S.A.R.

Aims The aims of this study were to explore the interrelationship between parenting stress, parental feeding and children eating behaviour and weight outcomes in order to tackle the rapid increased childhood obesity in Hong Kong.

Methods 336 Chinese parents of young children aged 2-7 years from 27 kindergartens were recruited for a cross sectional study. The Parental Feeding Style Questionnaire (PFSQ), Child Eating Behaviour Questionnaire (CEBO) and Parenting Stress Index (PSI$\mathrm{SF})$ were employed. Young children's BMI were classified by the IOTF. Path analyses were used for data analysis.

Results Several path models were attempted. The first model indicated that higher parenting stress significantly predicted higher children food responsiveness (Standardized $\beta$-coefficient $=0.195$, $p<0.001$ ) which predicted higher weight status (Standardized $\beta$-coefficient $=0.249, p<0.001)$. Higher parenting stress also predicted higher instrumental feeding (Standardized $\beta$-coefficient $=$ $0.294, p<0.001$ ) which predicted lower weight status (Standardized $\beta$-coefficient $=-0.204, p<0.001)$. Food responsiveness and instrumental feeding were weakly correlated $(r=0.288, p<0.001)$. The standardized indirect effects of parenting stress on children's weight status via food responsiveness (Standardized Sobel's $Z=2.799$, $\mathrm{p}<0.01$ ) and instrumental feeding (Standardized Sobel's $Z=-3.015$, $\mathrm{p}<0.01$ ) were significant. The second model showed higher parenting stress predicted higher emotional feeding (Standardized $\beta$-coefficient $=0.242, p<0.001)$ which predicted higher weight status (Standardized $\beta$-coefficient $=0.249, \mathrm{p}<0.001$ ).

Conclusions These findings could suggest directions to childhood obesity interventions.

\section{USING NEW CHILD GROWTH STANDARDS OF WORLD HEALTH ORGANIZATION FOR ESTIMATION OVERWEIGHT AND OBESITY IN PRE-SCHOOL CHILDREN, AHWAZ, IRAN}

doi:10.1136/archdischild-2012-302724.1440

${ }^{1} \mathrm{~S}$ Nouhjah, ${ }^{2} \mathrm{M}$ Karandish, ${ }^{3} \mathrm{R}$ Malihi. 'Social Determinants of Health Research Center, Department of Public Health, Ahvaz Jundishapur Medical Sciences University; ${ }^{2}$ Diabetes Research Center, Department of Nutrition; ${ }^{3}$ Department of Nutrition, Jundishapur Medical Sciences University, Ahvaz, Iran

Background and Aims Childhood obesity as a global health problem is associated with increased risk of mortality and morbidity. A few studies are focused on prevalence of children obesity based on new WHO growth standards in Iran. The aim of this study was to determine prevalence of overweight and obesity in children attending health centers based on new world health organization growth standards.

Methods In a descriptive study 1035 children aged 24-60 months, attending urban public health centers for receiving routine children health care were surveyed in 2009. Interview with mothers, filling a questionnaire by trained questioner were used for data collection. Soft ware of WHO for PL (WHO Anthro beta version, 17 February 2006) used for data analysis after entrancing data in SPSS software. Overweight and obesity was defined based on Body Mass Index/age above $+2 \mathrm{SD}$ and $+3 \mathrm{SD}$ of median $\mathrm{Z}$ score.

Results Overall, $15 \%$ of the children were overweight. Highest prevalence of overweight was observed in the 24-35 month age group (19.4\%).In different age groups of boys and girls, the prevalence of overweight and obesity was calculated $16.7 \%$ and $13.3 \%$ respectively. Overall prevalence of obesity was $7.3 \%$. Highest prevalence of obesity in the 24-35 month age group (10.4\%) was observed. Prevalence of obesity in boys and girls was $9 \%$ and $5.6 \%$ respectively.

Conclusion Overweight and obesity in $2-5$ years children has reached alarming proportion in Ahwaz. Urgent interventional health programs are needed to prevent childhood obesity. 

STATUS AMONG SOUTH SINAI CHILDREN

doi:10.1136/archdischild-2012-302724.1441

${ }^{1} \mathrm{NE}-\mathrm{M}$ Hassan, ${ }^{1} \mathrm{SA}$ El-Masry, ${ }^{2} \mathrm{GA}$ Yamamah. 'Biological Anthropology; ${ }^{2}$ Peadiatrics, National Research Centre, Cairo, Egypt

Background No information exists on nutritional status of South Sinai residing children, Egypt.

Aim Assessing prevalence of malnutrition among South Sinai children.

Methods Cross sectional study included 3987 healthy children (0 to 11 years); randomly selected; represent about $12 \%$ of all children from the 6 areas of South Sinai. Height and weight were measured. Weight-for-age Z score (WAZ), height-for-age Z score (HAZ) and weight-for-height $Z$ score (HAZ) were used to estimate the children's nutritional status. Venous blood sample was obtained to measure plasma hemoglobin level for school students.

Results Wasting (WHZ<-1.96 SD), underweight (WAZ $<-1.96$ $\mathrm{SD})$ and stunting (HAZ $<-1.96 \mathrm{SD}$ ) were prevalent among $4.2 \%$, $8.9 \%$ and $11 \%$, respectively. Prevalence of underweight, at risk of wasting and be anemic were more prevalent among boys than girls $(p<0.01)$. On the other side, $8 \%$ were overweight and $4 \%$ were obese. Although small percentage of anemic school children was suffering from growth deviation (wasting, 2.8\%; underweight, $5.6 \%$; stunting, $9.9 \%$; overweight, $2.8 \%$ and obese, $1.4 \%$ ), $.55 \%$ of them were at risk of growth deviations (wasting, underweight and stunting). Under nutrition were more prevalent among South Sinai children than their peers in Greater Cairo, while over nutrition was less prevalent.

Conclusion The highest prevalence of malnutrition was detected in infant's age. Anemia of primary school children was more prevalent among those at risk of under nutrition than undernourished ones.

Community education on environmental sanitation and personal hygienic practices, proper child rearing, breast-feeding and weaning practices would possibly reverse the trends.

\section{ELEMENTARY SCHOOL-BASED OBESITY PREVENTION INTERVENTION EFFECT ON WAIST CIRCUMFERENCE AMONG MULTIETHNIC 6-13 YEAR OLDS}

doi:10.1136/archdischild-2012-302724.1442

${ }^{1} \mathrm{D}$ Hollar, ${ }^{2} \mathrm{G}$ Lopez-Mitnik, ${ }^{3} \mathrm{~L}$ Hollar, ${ }^{2} \mathrm{~S}$ Messiah. ${ }^{1}$ Mississippi Food Network/University of Miami Miller School of Medicine; 'University of Miami Miller School of Medicine, Miami; ${ }^{3}$ Nova Southeastern University College of Osteopathic Medicine, Fort Lauderdale, FL, USA

Background Childhood onset obesity and related health consequences continue to be major clinical and public health issues in the USA and abroad. Schools provide an opportunity to implement prevention strategies to large, diverse pediatric audiences. Healthier Options for Public Schoolchildren (HOPS) was a school-based obesity prevention intervention with nutrition and physical activity components implemented in the elementary school setting targeting 6-13 year olds.

Methods HOPS was a quasi-experimental elementary schoolbased obesity prevention intervention targeting ethnically diverse 6-13-year-olds (Kindergarten-6 ${ }^{\text {th }}$ ). Over four school years (August 2004-June 2009), five schools (four intervention; one control, $\mathrm{N}=3,183,48 \%$ Hispanic) in Florida participated in the study. Waist circumference (WC) data was reported in the Fall of 2005 and Spring of 2006 only and these one year results are reported here.

Results Among boys, the mean incremental change in WC (measured in centimeters $[\mathrm{cm}]$ ) increase was significantly less in the intervention $(1.35 \mathrm{~cm}+/-0.88$ [SD]) versus control schools $(3.83 \mathrm{~cm}$ $+/-0.94)(\mathrm{P}<0.0001)$. Among girls the mean incremental change in
WC increase was significantly less in the intervention $(1.20 \mathrm{~cm}+/-$ $0.84)$ versus control schools $(4.17 \mathrm{~cm}+/-0.89)(\mathrm{P}<0.0001)$. Similarly, waist-to-height ratio results showed that the intervention group mean incremental change was significantly less versus the control group for boys $(\mathrm{P}=0.0002)$ and girls $(\mathrm{P}<0.0001)$.

Conclusions Elevated WC is strongly correlated with cardiometabolic disease risk factors and should be monitored in young children as such. School-based obesity prevention interventions show promise in improving weight and potentially cardiometabolic health in elementary-school aged children.

\section{RELATION OF BIRTH WEIGHT AND INFANT GROWTH WITH BODY SIZE, BODY COMPOSITION AND FAT DISTRIBUTION AT AGE 5-6 YEARS}

doi:10.1136/archdischild-2012-302724.1443

${ }^{1} \mathrm{M}$ De Beer, ${ }^{2} \mathrm{M}$ Van Eijsden, ${ }^{3} \mathrm{~T}$ Vrijkotte, ${ }^{4} \mathrm{C}$ Fall, ${ }^{4} \mathrm{C}$ Osmond, ${ }^{1} \mathrm{R}$ Gemke. ${ }^{1}$ Pediatrics, VU University Medical Center; ${ }^{2} E$ Eidemiology, Documentation and Health Promotion, Municipal Health Service; ${ }^{3}$ Social Medicine, Academic Medical Center, Amsterdam, The Netherlands; ${ }^{4}$ MRC Lifecourse Epidemiology Unit, Southampton General Hospital, Southampton, UK

Background and Aims In a prospective cohort study (the $A B C D$ study), we investigated the association of early growth with (precursors of) childhood obesity.

Methods Our study was based on 2,338 term born children with on average 7 measures of growth (weight and length) from birth to age 12 months. We used conditional weight, a residual of current weight regressed on prior weights, to represent deviations from expected weight gain from 0 to 1,1 to 3,3 to 6 , and 6 to 12 months. The same method was applied for conditional length and body mass index. Relations of these measures with height, body mass index, fat mass (adjusted for height and fat free mass) and waist-to-height ratio (WHtR) at age 5-6 were explored using linear regression analysis.

Results Higher birth weight, weigh conditionals and bmi conditionals for all periods in infancy (expressing faster growth) were associated with increased height, body mass index, fat mass and WHtR. Furthermore, although higher length conditionals after 3 months were associated with higher childhood bmi, no association was found with fat mass. Higher length conditionals were associated with lower WHtR.

Conclusions Faster prenatal growth and subsequent faster infant weight- and bmi gain were associated with greater childhood body size, more fat tissue mass and more central adipose distribution. Conversely, although rapid length gain after 3 months was associated with greater childhood body size, this was not accompanied by more fat tissue mass, yet with less central adipose distribution.

\section{ASSESSMENT OF ALIMENTARY BEHAVIOR AND PHYSICAL ACTIVITY IN ROMANIAN OBESE SCHOOL CHILDREN}

doi:10.1136/archdischild-2012-302724.1444

SC Cosoveanu, IO Petrescu, I Puiu. 2nd Pediatric Clinic, Emergency County Hospital, University of Medicine and Pharmacy Craiova, Craiova, Romania

Background and Aims Obesity represents a major, global health problem, continuously increasing all over the world, including Romania. We carried out an epidemiological study on the alimentary behavior and physical activity in overweight and obese school children.

Methods The target population was represented by 41 overweight school children, 68 obese and 57 normal weight, from Craiova, in 2008-2011. Inclusion criteria: children aged 6-14 years with BMI $\geq 95$ percentile/sex/age for obesity, $85 \leq \mathrm{BMI}<95$ percentile/sex/age for overweight and $85<\mathrm{IMC} \leq 5$ percentile/sex/age for normal weight. For every child in our group we followed the alimentary inquiry, the 